\title{
A study of the inextensible flows of tube-like surfaces associated with focal curves in Galilean 3-space $G_{3}$
}

\author{
Adel H. Sorour
}

\begin{abstract}
In this paper, we study inextensible flows of focal curves associated with tube-like surfaces in Galilean 3 -space $G_{3}$. We give some characterizations for curvature and torsion of focal curves associated with tube-like surfaces in Galilean 3-space $G_{3}$. Furthermore, we show that if flow of this tube-like surface is inextensible then this surface is not developable as well as not minimal. Finally an example of tube-like surface is used to demonstrate our theoretical results and graphed.
\end{abstract}

Mathematics Subject Classification (2010): 31B30, 58E20.

Keywords: Tube-like surface, Gaussian curvature, mean curvature, Galilean 3space.

\section{Introduction}

Curve design using splines is one of the most fundamental topics in CAGD. Inextensible flows of curves possess a beautiful shape preserving connection to their control polygon. They allow us the formulation of algorithms for processing, especially subdivision algorithms. Moreover, at least the curves of odd degree and maximal smoothness also arise as solutions of variational problems.

In the past two decades, for the need to explain certain physical phenomena and to solve practical problems, geometers and geometric analysis have begun to deal with curves and surfaces which are subject to various forces and which flow or evolve with time in response to those forces so that the metrics are changing. Now, various geometric flows have become one of the central topics in geometric analysis. Many authors have studied geometric flow problems. In [9, 10] Kwon et al. studied inextensible flows of curves and developable surface in $\mathbb{R}^{3}$.

Korpinar et al. [8] studied inextensible flows of developable surfaces associated focal curve of helices in Euclidean 3 -space $E^{3}$. Differential geometry of the Galilean 
space $G_{3}$ has been largely developed in Kamenarovic [6], Ogrenmiş et al. [13, 14] and Roschel [17].

In this work, we study inextensible flows of focal curves associated with tubelike surfaces in Galilean 3-space $G_{3}$. We give some characterizations for curvature and torsion of focal curves associated with tube-like surfaces in Galilean 3-space $G_{3}$. Finally, we show that if the flow of a tube-like surface associated to a focal curve is inextensible, then the surface is not developable as well as not minimal for an arbitrary focal curve. We used some idea from Korpinar et al. [8] in this paper.

\section{Preliminaries}

The geometry of the Galilean space $G_{3}$ has been treated in detail in O. Roschl's habilitation in 1984 [17]. More about Galilean space and Pseudo-Galilean space may be found in $[20,1,3,7,11,12,21]$. The Galilean space $G_{3}$ is a Cayley-Klein space equipped with the projective metric of signature $(0,0,+,+)$, as in [21].

The Galilean space is a three dimensional complex projective space $P_{3}$ in which the absolute figure $\left\{\omega, f, I_{1}, I_{2}\right\}$ consists of a real plane $\omega$ (the absolute plane), a real line $f \subset \omega$ (the absolute line) and two complex conjugate points $I_{1}, I_{2} \in f$ (the absolute points) [6]. We shall take, as a real model of the space $G_{3}$, a real projective space $P_{3}$ with the absolute $\{\omega, f\}$ consisting of a real plane $\omega \subset G_{3}$ and a real line $f \in \omega$ on which an elliptic involution $\varepsilon$ has been defined. In homogeneous coordinates

$$
\begin{aligned}
& \omega \ldots x_{0}=0, \quad f \ldots x_{0}=x_{1}=0, \\
& \varepsilon:\left(0: 0: x_{2}: x_{3}\right) \rightarrow\left(0: 0: x_{3}:-x_{2}\right),
\end{aligned}
$$

while in the nonhomogeneous coordinates, the similarity group $H_{8}$ has the form

$$
\begin{gathered}
\bar{x}=a_{11}+a_{12} x, \\
\bar{y}=a_{21}+a_{22} x+a_{23}(y \cos [\phi]+z \sin [\phi]), \\
\bar{z}=a_{31}+a_{32} x-a_{33}(y \sin [\phi]-z \cos [\phi]),
\end{gathered}
$$

where $a_{i j}$ and $\phi$ are real numbers. For $a_{12}=a_{23}=1$, we have the subgroup $B_{6}$ which is the group of Galilean motions:

$$
\begin{aligned}
& \bar{x}=a+x, \\
& \bar{y}=b+c x+y \cos [\phi]+z \sin [\phi], \\
& \bar{z}=d+e x-y \sin [\phi]+z \cos [\phi] .
\end{aligned}
$$

It is worth noting that [16]: in $G_{3}$ there are four classes of lines:

a): (proper) nonisotropic lines: they do not meet the absolute line $f$.

b): (proper) isotropic lines: lines that do not belong to the plane $\omega$ but meet the absolute line $f$.

c): (unproper) nonisotropic lines: all lines of $\omega$ but $f$.

d): the absolute line $f$.

Planes $x=$ constant are Euclidean and so is the plane $\omega$. Other planes are isotropic. In what follows, the coefficients $a_{12}$ and $a_{23}$ will play a special role. 
In particular, for $a_{12}=a_{23}=1,(2.2)$ defines the group $B_{6} \subset H_{8}$ of isometries of the Galilean space $G_{3}$.

In affine coordinates, the Galilean scalar product between two vectors $a=$ $\left(a_{1}, a_{2}, a_{3}\right)$ and $b=\left(b_{1}, b_{2}, b_{3}\right)$ is defined by [15]

$$
(\langle a, b\rangle)_{G_{3}}= \begin{cases}a_{1} b_{1}, & \text { if } a_{1} \neq 0 \text { or } b_{1} \neq 0, \\ a_{2} b_{2}+a_{3} b_{3}, & \text { if } a_{1}=0 \text { and } b_{1}=0 .\end{cases}
$$

The Galilean cross product is defined by

$$
(a \wedge b)_{G_{3}}=\left\{\begin{array}{l}
\left|\begin{array}{ccc}
0 & e_{2} & e_{3} \\
a_{1} & a_{2} & a_{3} \\
b_{1} & b_{2} & b_{3}
\end{array}\right|, \text { if } a_{1} \neq 0 \text { or } b_{1} \neq 0, \\
\left|\begin{array}{ccc}
e_{1} & e_{2} & e_{3} \\
a_{1} & a_{2} & a_{3} \\
b_{1} & b_{2} & b_{3}
\end{array}\right|, \text { if } a_{1}=0 \text { and } b_{1}=0 .
\end{array}\right.
$$

The unit Galilean sphere is defined by [5]

$$
S_{ \pm}^{2}=\left\{\alpha \in G_{3} \mid\langle\alpha, \alpha\rangle_{G_{3}}=\mp r^{2}\right\} .
$$

Let $M: \Phi=\Phi(u, v)$ be a surface in Galilean 3-space is given by the parametrization

$$
\Phi(u, v)=(x(u, v), y(u, v), z(u, v)), \quad u, v \in \mathbb{R},
$$

where $x(u, v), y(u, v), z(u, v) \in C^{3}$. The isotropic unit normal vector $U$ of the surface $M$ is defined by

$$
U(u, v)=\frac{\Phi_{u} \wedge \Phi_{v}}{\left\|\Phi_{u} \wedge \Phi_{v}\right\|}, \quad \Phi_{u}=\frac{\partial \Phi}{\partial u}, \quad \Phi_{v}=\frac{\partial \Phi}{\partial v}
$$

or equivalently

$$
U(u, v)=\frac{\left(0, x_{v} z_{u}-x_{u} z_{v}, x_{u} y_{v}-x_{v} y_{u}\right)}{\sqrt{\left(x_{v} z_{u}-x_{u} z_{v}\right)^{2}+\left(x_{u} y_{v}-x_{v} y_{u}\right)^{2}}},
$$

where $x_{u}=\frac{\partial x(u, v)}{\partial u}, x_{v}=\frac{\partial x(u, v)}{\partial v}$. Using (2.1) and $W=\left\|\Phi_{u} \wedge \Phi_{v}\right\|$, we get the isotropic unit vector $\delta(u, v)$ in the tangent plane of the surface as [4]

$$
\delta(u, v)=\frac{\left(0, x_{v} y_{u}-x_{u} y_{v}, x_{v} z_{u}-x_{u} z_{v}\right)}{W},
$$

where

$$
\langle\delta, \delta\rangle=1, \quad\langle U, \delta\rangle=0,
$$

by means of Galilean geometry. Observe that a straightforward computation shows that $\delta$ can be expressed by

$$
\delta(u, v)=\frac{x_{v} \Phi_{u}-x_{u} \Phi_{v}}{W}
$$


The first fundamental form $d s^{2}$ of a surface $M$ in $G_{3}$ is given by

$$
\begin{aligned}
I & =d s^{2} \\
& =\left(g_{1} d u+g_{2} d v\right)^{2}+\epsilon\left(h_{11} d u^{2}+2 h_{12} d u d v+h_{22} d v^{2}\right)
\end{aligned}
$$

where

$$
\begin{aligned}
& g_{1}=x_{u}, \quad g_{2}=x_{v}, \quad g_{i j}=g_{i} g_{j}, \\
& h_{11}=\left\langle\Phi_{u}, \Phi_{u}\right\rangle, h_{12}=\left\langle\Phi_{u}, \Phi_{v}\right\rangle, h_{22}=\left\langle\Phi_{v}, \Phi_{v}\right\rangle,
\end{aligned}
$$

and

$$
\epsilon=\left\{\begin{array}{l}
0, \text { if direction } d u: d v \text { is non-isotropic, } \\
1, \text { if direction } d u: d v \text { is isotropic. }
\end{array}\right.
$$

The coefficients of the second fundamental form can be determined from

$$
L_{i j}=\left\langle\frac{\Phi_{i j} x_{u}-x_{i j} \Phi_{u}}{x_{u}}, U\right\rangle=\left\langle\frac{\Phi_{i j} x_{v}-x_{i j} \Phi_{v}}{x_{v}}, U\right\rangle,
$$

where $\Phi_{i j}$ denotes the second order partial differentials of $M$ and the indices $i, j$ belong to the parameters $u, v$ respectively. Under this parametrization of the surface $M$, the Gaussian curvature $K$ and the mean curvature $H$ have the classical expressions, respectively [11]

$$
\begin{gathered}
K=\frac{\operatorname{det}\left(L_{i j}\right)}{W^{2}}=\frac{L_{11} L_{22}-L_{12}^{2}}{h_{11} h_{22}-h_{12}^{2}} \\
H=\frac{1}{2} h^{i j} L_{i j}=\frac{h_{11} L_{22}+h_{22} L_{11}-2 h_{12} L_{12}}{2\left(h_{11} h_{22}-h_{12}^{2}\right)} .
\end{gathered}
$$

\section{Inextensible flows of tube-like surfaces associated with focal curves in $G_{3}$}

The aim of this section, we will obtain the tube-like surface from the tube surface. Since the tube surfaces are special kinds of the canal surfaces in Galilean 3-space.

If we find the canal surface with taking variable radius $r(u)$ as constant, then the tube surface can be found, since the canal surface is a general case of the tube surface. An envelope of a 1-parameter family of surfaces is constructed in the same way that we constructed a 1-parameter family of curves. The family is described by a differentiable function $F(x, y, z, \lambda)=0$, where $\lambda$ is a parameter. When $\lambda$ can be eliminated from the equations

$$
F(x, y, z, \lambda)=0
$$

and

$$
\frac{\partial F(x, y, z, \lambda)}{\partial \lambda}=0
$$

we get the envelope, which is a surface described implicitly as $G(x, y, z)=0$. For example, for a 1-parameter family of planes we get a developable surface [18]. 
Definition 3.1. The envelope of a 1-parameter family $u \rightarrow S_{ \pm}^{2}$ of the spheres in $G_{3}$ is called a canal surface in Galilean 3-space. The curve formed by the centers of the Galilean spheres is called center curve of the canal surface.The radius of the canal surface is the function $\mathrm{r}$ such that $r(u)$ is the radius of the Galilean sphere $S_{ \pm}^{2}$. Then, the canal surface can be parametrized as follows

$$
C(u, v)=\alpha(u)+r(u)(\cos [v] N(u)+\sin [v] B(u)) .
$$

Definition 3.2. Let $\alpha:(a, b) \rightarrow G_{3}$ be a unit speed curve whose curvature does not vanish. Consider a tube of radius $r$ around $\alpha$. Since the normal $N$ and binormal $B$ are perpendicular to $\alpha$, the Galilean circle is perpendicular $\alpha$ and $\alpha(u)$. As this Galilean circle moves along $\alpha$, it traces out a surface about $\alpha$ which will be the tube about $\alpha$, provided $r$ is not too large. If the radius function $r(u)=r$ is a constant, then, the canal surface is called a tube (pipe) surface and it parametrized as

$$
\operatorname{Tube}(u, v)=\alpha(u)+r(\cos [v] N(u)+\sin [v] B(u)) .
$$

Theorem 3.3. Let $\alpha: I \rightarrow G_{3}$ be a curve in Galilean 3-space. Assume the center curve of a tube-like surface is a unit speed curve $\alpha$ with nonzero curvature. Then, the tube-like surface can be expressed as follows

$$
X(u, v)=\alpha(v)+r(\cos [u] N(v)-\sin [u] B(v)),
$$

where $T, N$ and $B$ are the tangent, principal normal and binormal of $\alpha$.

Proof. Suppose $X$ is a patch that parametrizes the envelope of the Galilean spheres defining tube-like surface. Where the curvature of $\alpha(v)$ is nonzero, the Frenet frame of it is well defined, and we can write

$$
X(u, v)-\alpha(v)=p(u, v) T(v)+q(u, v) N(v)-w(u, v) B(v),
$$

where $p, q$ and $w$ are differentiable on the interval on which $\alpha$ is defined. We have

$$
\langle X(u, v)-\alpha(v), X(u, v)-\alpha(v)\rangle_{G_{3}}=\left\{\begin{array}{l}
p^{2}=r^{2} \text { if } p(u, v) \neq 0, \\
q^{2}+w^{2}=r^{2} \text { if } p(u, v)=0 .
\end{array}\right.
$$

The equation (3.5) expresses analytically the geometric fact that $X(u, v)$ lies on a Galilean sphere $S_{ \pm}^{2}(v)$ of radius $r$ centrered at $\alpha(v)$. Furthermore, $X(u, v)-\alpha(v)$ is a normal vector to the tube-like surface; this fact implies that

$$
\begin{aligned}
& \left\langle X(u, v)-\alpha(v), X_{u}\right\rangle_{G_{3}}=0, \\
& \left\langle X(u, v)-\alpha(v), X_{v}\right\rangle_{G_{3}}=0 .
\end{aligned}
$$

Equations (3.5), (3.6) and (3.7) say that the vectors $X_{u}$ and $X_{v}$ are tangents to $S_{ \pm}^{2}(v)$. Calculating the partial derivative of (3.4) with respect to $u$ and $v$ respectively, we obtain

$$
\begin{gathered}
X_{u}=p_{u} T+q_{u} N-w_{u} B \\
X_{v}=\left(1+p_{v}\right) T+\left(p \kappa+q_{v}+w \tau\right) N+\left(q \tau-w_{v}\right) B .
\end{gathered}
$$


Case 1. If $p(u, v) \neq 0$, from (3.4) and (3.5), we have

$$
\left\{\begin{array}{ccc}
p^{2} & =r^{2} \\
p p_{v} & =0
\end{array}\right.
$$

Equations (3.5), (3.7), (3.9) and (3.10) imply

$$
\left(1+p_{v}\right) p=0 \text {. }
$$

From (3.10) and (3.11), we get

$$
r=0 .
$$

Hence, the equation (3.4) is not surface

Case 2. If $p(u, v)=0$. From (3.4) and (3.5), we have the following

$$
\left\{\begin{array}{l}
q^{2}+w^{2}=r^{2} \\
q q_{v}+w w_{v}=0(r=\text { constant }) .
\end{array}\right.
$$

Then, Eqs. (3.6), (3.8) and (3.13) imply that

$$
q q_{u}+w w_{u}=0(r=\text { constant }) .
$$

From (3.13) and (3.14), we obtain

$$
\left\{\begin{array}{lll}
q & = & r \cos [u] \\
w & = & r \sin [u]
\end{array}\right.
$$

Thus, (3.4) becomes

$$
X(u, v)=\alpha(v)+r(\cos [u] N(v)-\sin [u] B(v)) .
$$

From the above theorem, one can formulate the following definition:

Definition 3.4. Given a space curve $\alpha(v)=(x(v), y(v), z(v))$, at each point, there are three directions associated with it, the tangent, normal and binormal directions. The unit tangent vector is denoted by $T$, i.e., $T(v)=\frac{\alpha^{\prime}(v)}{\left\|\alpha^{\prime}(v)\right\|}$, the unit normal vector is denoted by $N$, i.e., $N(v)=\frac{T^{\prime}(v)}{\left\|T^{\prime}(v)\right\|}$, the unit binormal vector is denoted by $B$, i.e., $B(v)=T(v) \wedge N(v)$ (cross product). With $\alpha(v), T(v), N(v)$ and $B(v)$, a tube-like surface can be expressed as follows [19]

$$
M: X(u, v)=\alpha(v)+r(\cos [u] N(v)-\sin [u] B(v)),
$$

where $r$ is a parameter corresponding to the radius of the rotation (In general $r$ can be a function of $v$ ). For fixed $v$, when $u$ runs from 0 to $2 \pi$, we have a circle around the point $\alpha(v)$ in the $T, N$ plane. As we change $v$, this circle moves along the space curve $\alpha$, and we will generate a tube-like surface along $\alpha$ (a special kind of tube surfaces defined by (3.16)).

Let $\alpha: I \subset \mathbb{R} \rightarrow G_{3}$, be an unit speed curve in Galilean space $G_{3}$ given by

$$
\alpha(v)=(v, y(v), z(v))
$$


where $v$ is a Galilean invariant parameter (the arc-length on $\alpha$ ). The orthonormal frame in the sense of Galilean space $G_{3}$ is defined by

$$
\left\{\begin{array}{l}
T(v)=\alpha^{\prime}(v)=\left(1, y^{\prime}(v), z^{\prime}(v)\right), \\
N(v)=\frac{\alpha^{\prime \prime}(v)}{\kappa(v)}=\frac{1}{\kappa(v)}\left(0, y^{\prime \prime}(v), z^{\prime \prime}(v)\right), \\
B(v)=(T(v) \wedge N(v))_{G}=\frac{1}{\kappa(v)}\left(0,-z^{\prime \prime}(v), y^{\prime \prime}(v)\right),
\end{array}\right.
$$

where $\kappa(v)=\left\|\alpha^{\prime \prime}(v)\right\|=\sqrt{\left(y^{\prime \prime}(v)\right)^{2}+\left(z^{\prime \prime}(v)\right)^{2}}$ is the curvature and

$$
\tau(v)=\frac{1}{\kappa^{2}(v)} \operatorname{det}\left[\alpha^{\prime}(v), \alpha^{\prime \prime}(v), \alpha^{\prime \prime \prime}(v)\right]
$$

is the torsion. The vectors $T(v), N(v)$ and $B(v)$ in (3.18) are called of the tangent vector, the principal normal vector and the binormal vector of $\alpha(v)$, respectively. They satisfy the following Frenet equations [13]

$$
\left[\begin{array}{c}
T^{\prime}(v) \\
N^{\prime}(v) \\
B^{\prime}(v)
\end{array}\right]=\left[\begin{array}{ccc}
0 & \kappa(v) & 0 \\
0 & 0 & \tau(v) \\
0 & -\tau(v) & 0
\end{array}\right]\left[\begin{array}{c}
T(v) \\
N(v) \\
B(v)
\end{array}\right],
$$

where the prime denotes the differentiation with respect to $v$ and we denote by $\kappa, \tau$ the curvature and the torsion of the curve $\alpha$. We can know that $T, N, B$ are mutually orthogonal vector fields satisfying equations

$$
\begin{aligned}
& \langle T, T\rangle_{G}=\langle N, N\rangle_{G}=\langle B, B\rangle_{G}=1, \\
& \langle T, N\rangle_{G}=\langle T, B\rangle_{G}=\langle N, B\rangle_{G}=0, \\
& \operatorname{det}(T, N, B)_{G}=1 .
\end{aligned}
$$

Using the equations (3.16), (3.17) and (3.18), we have

$$
X(u, v)=(v, y(v), z(v))+\frac{r}{\kappa}\left[\left(0, y^{\prime \prime}(v), z^{\prime \prime}(v)\right) \cos [u]-\left(0,-z^{\prime \prime}(v), y^{\prime \prime}(v)\right) \sin [u]\right]
$$

From now on, For a unit speed curve $\alpha=\alpha(v): I \rightarrow G_{3}$, the curve consisting of the centers of the osculating spheres of $\alpha$ is called the parametrized focal curve of $\alpha$. The hyperplanes normal to $\alpha$ at a point consist of the set of centers of all spheres tangent to $\alpha$ at that point. Hence the center of the osculating spheres at that point lies in such a normal plane. Therefore, denoting the focal curve by $C_{\alpha}$, we can write [2]

$$
C_{\alpha}(v)=\left(\alpha+c_{1} N+c_{2} B\right)(v)
$$

where the coefficients $c_{1}, c_{2}$ are smooth functions of the parameter of the curve $\alpha$, called the first and second focal curvatures of $\alpha$, respectively. Further, the focal curvatures $c_{1}, c_{2}$ are defined by

$$
c_{1}=\frac{1}{\kappa}, \quad c_{2}=\frac{c_{1}^{\prime}}{\tau}, \quad \kappa \neq 0, \quad \tau \neq 0 .
$$


Lemma 3.5. Let $\alpha: I \rightarrow G_{3}$ be a unit speed helix and $C_{\alpha}$ its focal curve on $G_{3}$. Then,

$$
c_{1}=\frac{1}{\kappa}=\text { constant and } c_{2}=0 \text {. }
$$

On the other hand, the fundamental quantities $h_{i j}, L_{i j}$ and its evolution of tubelike surface (3.16) are obtained, respectively. Thus the Gaussian, mean curvatures and its evolution of such surface are given. For this purpose, let a tube-like surface generated by sweeping a space curve along another central space curve, moving in 3-dimensional Galilean space $G_{3}$, be given at time $t$ by the parametrization

$$
\left\{\begin{array}{l}
\bar{X}(u, v, t)=C_{\alpha}(v, t)+r(\cos [u, t] N(v, t)-\sin [u, t] B(v, t)), \\
\text { where } \bar{X}(u, v, 0)=X(u, v), C_{\alpha}(v, 0)=C_{\alpha}(v), \cos [u, 0]=\cos [u], \\
N(v, 0)=N(v), \sin [u, 0]=\sin [u] \text { and } B(v, 0)=B(v) .
\end{array}\right.
$$

Definition 3.6. A smooth surface $X(u, v)$ is called a developable surface if its Gaussian curvature $K$ vanishes everywhere on the surface.

Definition 3.7. [10] A surface evolution $\bar{X}(u, v, t)$ and its flow $\frac{\partial \bar{X}}{\partial t}$ are said to be inextensible if its coefficients first fundamental form $\left\{h_{11}, h_{12}, h_{22}\right\}$ satisfies

$$
\frac{\partial h_{11}}{\partial t}=\frac{\partial h_{12}}{\partial t}=\frac{\partial h_{22}}{\partial t}=0 .
$$

This definition states that the surface $\bar{X}(u, v, t)$ is, for all time $t$, the isometric image of the original surface $\bar{X}\left(u, v, t_{0}\right)$ defined at some initial time $t_{0}$. For a tube-like surface, $\bar{X}(u, v, t)$ can be physically pictured as the parametrization of a waving flag. For a given surface that is rigid, there exists no nontrivial inextensible evolution.

Theorem 3.8. Let $\bar{X}$ be the tube-like surface associated with focal curve in $G_{3} \cdot \frac{\partial \bar{X}}{\partial t}$ is inextensible, then

$$
\frac{\partial \bar{X}}{\partial t}=0
$$

Proof. Suppose that $\bar{X}(u, v, t)$ be a tube-like surface. We show that $\bar{X}$ is inextensible.

$$
\begin{aligned}
& \bar{X}_{u}=-r[\sin [u, t] N+\cos [u, t] B], \\
& \bar{X}_{v}=T+r \tau \sin [u, t] N+\left[c_{1} \tau+c_{2}^{\prime}+r \tau \cos [u, t]\right] B .
\end{aligned}
$$

Equations (2.9) and (3.27) lead to the coefficients of the first fundamental form obtained by

$$
h_{11}=r^{2}, \quad h_{12}=0, \quad h_{22}=1 .
$$

Under the previous calculations, we have

$$
\frac{\partial h_{11}}{\partial t}=0, \quad \frac{\partial h_{12}}{\partial t}=0, \quad \frac{\partial h_{22}}{\partial t}=0 .
$$

If $\frac{\partial \bar{X}}{\partial t}$ is inextensible, then we have (3.26). 
Theorem 3.9. Let $\bar{X}(u, v, t)$ be the tube-like surface associated with focal curve in $G_{3}$. If flow of this tube-like surface is inextensible then this surface is not developable as well as not minimal.

Proof. Assume that $\bar{X}$ be a tube-like surface parametrized by (3.24). The vector cross product of $\bar{X}_{u}$ and $\bar{X}_{v}$ is given by

$$
\bar{X}_{u} \wedge \bar{X}_{v}=-r[\cos [u, t] N-\sin [u, t] B] .
$$

Hence, one can get

$$
\left\|\bar{X}_{u} \wedge \bar{X}_{v}\right\|=r
$$

Using equations (3.29) and (3.30), we obtain the isotropic normal vector of tube-like surface as

$$
U=\frac{\bar{X}_{u} \wedge \bar{X}_{v}}{\left\|\bar{X}_{u} \wedge \bar{X}_{v}\right\|}=-\cos [u, t] N+\sin [u, t] B .
$$

The second order partial differentials of $\bar{X}$ are found

$$
\left\{\begin{aligned}
\bar{X}_{u u}= & r[-\cos [u, t] N+\sin [u, t] B] \\
\bar{X}_{u v}= & r \tau[\cos [u, t] N-\sin [u, t] B] \\
\bar{X}_{v v}= & {\left[\kappa+r \tau^{\prime} \sin [u, t]-c_{1} \tau^{2}-c_{2}^{\prime} \tau-r \tau^{2} \cos [u, t]\right] N+} \\
& {\left[r \tau^{2} \sin [u, t]+c_{1}^{\prime} \tau+c_{1} \tau^{\prime}+c_{2}^{\prime \prime}+r \tau^{\prime} \cos [u, t]\right] B . }
\end{aligned}\right.
$$

From the equations (3.31) and (3.32), one can compute the coefficients of the second fundamental form for the surface (3.24) as the following

$$
\left\{\begin{aligned}
L_{11}= & r \\
L_{12}= & -r \tau \\
L_{22}= & {\left[-\kappa+c_{1} \tau^{2}+c_{2}^{\prime} \tau\right] \cos [u, t]+} \\
& {\left[c_{1}^{\prime} \tau+c_{1} \tau^{\prime}+c_{2}^{\prime \prime}\right] \sin [u, t]+r \tau^{2} . }
\end{aligned}\right.
$$

Based on the above calculations, the Gaussian curvature $K$ and the mean curvature $H$ of (3.24) are given by, respectively

$$
\begin{gathered}
K=\frac{1}{r}\left(\left[-\kappa+c_{1} \tau^{2}+c_{2}^{\prime} \tau\right] \cos [u, t]+\left[c_{1}^{\prime} \tau+c_{1} \tau^{\prime}+c_{2}^{\prime \prime}\right] \sin [u, t]\right), \\
H=\frac{1}{2}\left(\left[-\kappa+c_{1} \tau^{2}+c_{2}^{\prime} \tau\right] \cos [u, t]+\left[c_{1}^{\prime} \tau+c_{1} \tau^{\prime}+c_{2}^{\prime \prime}\right] \sin [u, t]+r \tau^{2}\right)+\frac{1}{2 r} .
\end{gathered}
$$

By the use of (3.22) and above equations the proof is complete.

Here, we compute in special case the curvatures of the surface (3.24) as well as the curvatures associated to the focal curve of helix on this surface as follows:

At $\kappa=1, \tau=1$, the surface (3.24) has the following

$$
K=0, \quad H=\frac{r^{2}+1}{2 r} .
$$

Making use of the data described above, one can formulate the following theorem: 
Theorem 3.10. Let $\bar{X}(u, v, t)$ be a tube-like surface associated with focal curve of helix in $G_{3}$. If flow of this surface is inextensible then this surface is developable as well as not minimal.

\section{Applications}

In this section, we consider an example to illustrate the main results that we have presented in our paper.

Example 4.1. Let us consider a surface

$$
\bar{X}(u, v, t)=C_{\alpha}(v, t)+r(\cos [u, t] N(v, t)-\sin [u, t] B(v, t)),
$$

where $\alpha(v)$ is a helix

$$
\alpha(v)=(v, \cos [v], \sin [v]),
$$

it is easy to see that the Frenet's frame is

$$
\left\{\begin{array}{l}
T(v)=(1,-\sin [v], \cos [v]), \\
N(v)=(0,-\cos [v],-\sin [v]), \\
B(v)=(0, \sin [v],-\cos [v]) .
\end{array}\right.
$$

Since $\kappa=1$ is the curvature and $\tau=1$ is the torsion of the curve $\alpha$. Then, the focal curve of helix takes the form

$$
C_{\alpha}=(v, 0,0) .
$$

Thus, the surface (4.1) takes the following form

$\bar{X}(u, v, t)=(v,-r \cos [u, t] \cos [v, t]-r \sin [u, t] \sin [v, t], r \sin [u, t] \cos [v, t]-r \cos [u, t] \sin [v, t])$.

Calculating the partial derivative of (4.2) with respect to $u$ and $v$ respectively, we get $\bar{X}_{u}=(0, r \sin [u, t] \cos [v, t]-r \cos [u, t] \sin [v, t], r \cos [u, t] \cos [v, t]+r \sin [u, t] \sin [v, t])$, $\bar{X}_{v}=(1, r \cos [u, t] \sin [v, t]-r \sin [u, t] \cos [v, t],-r \sin [u, t] \sin [v, t]-r \cos [u, t] \cos [v, t])$. The components of the first and second fundamental forms of the surface (4.2) are given by, respectively

$$
h_{11}=r^{2}, \quad h_{12}=0, \quad h_{22}=1, \quad L_{11}=r, \quad L_{12}=-r, \quad L_{22}=r .
$$

The unit normal vector of the surface (4.2) takes the form

$$
U=(0, \sin [u, t] \sin [v, t]+\cos [u, t] \cos [v, t],-\sin [u, t] \cos [v, t]+\cos [u, t] \sin [v, t]) .
$$

For this surface, the Gaussian curvature $K$ and the mean curvature $H$ are defined by, respectively

$$
\begin{gathered}
K=0, \\
H=\frac{r^{2}+1}{2 r} .
\end{gathered}
$$


Then, the surface (4.2) is a developable and not minimal. One can see the graph of $\bar{X}(u, v, t)$ in Figure 1.

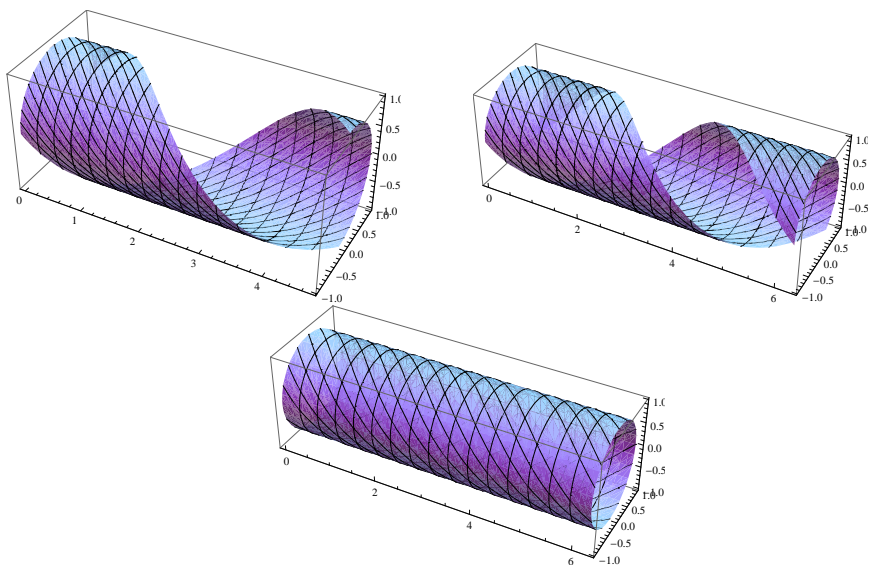

Figure 1. Some tube-like surfaces associated with focal curve of helices with $r=1, t=0$, Left: $u \in[0, \pi], v \in\left[0, \frac{3}{2} \pi\right]$, Middle: $u \in\left[0, \frac{13}{10} \pi\right], v \in[0,2 \pi]$ and Right: $u \in[0,2 \pi], v \in[0,2 \pi]$.

\section{References}

[1] Abdel-Aziz, H.S., Khalifa Saad, M., Weingarten timelike tube surfaces around a spacelike curve, Int. J. Math. Anal., 5(2011), 1225-1236.

[2] Alegre, P., Arslan, K., Carriazo, A., Murathan, C., Öztürk, G., Some special types of developable ruled surface, Hacettepe Journal of Mathematics and Statistics, 9(2010), no. 3, 319-325.

[3] Blaga, P.A., On tubular surfaces in computer graphics, Stud. Univ. Babeş-Bolyai Inform., $\mathbf{5 0}(2005), 81-90$.

[4] Dede, M., Tubular surfaces in Galilean space, Math. Commun., 18(2013), 209-217.

[5] Ergut, M., Ogrenmis, A.O., Some characterizations of a spherical curves in Galilean space $G_{3}$, Journal of Advanced Research in Pure Mathematics, 1-2(2009),18-26.

[6] Kamenarovic, I., Existence theorems for ruled surfaces in the Galilean space $G_{3}$, Rad Hazu Math., 456(1991), no. 10, 183-196.

[7] Karacan, M.K., Yayli, Y., On the geodesics of tubular surfaces in Minkowski 3-Space, Bull. Malays. Math. Sci. Soc., 31(2008), 1-10.

[8] Korpinar, T., Turhan, E., Altay, G., Inextensible flows of developable surfaces associated focal curve of helices in Euclidean 3-space $E^{3}$, Acta Univ. Apulensis, 29(2012), 235-240.

[9] Kwon, D.Y., Park, F.C., Evolution of inelastic plane curves, Appl. Math. Lett., 12(1999), 115-119.

[10] Kwon, D.Y., Park, F.C., Chi, D.P., Inextensible flows of curves and developable surfaces, Appl. Math. Lett., 18(2005), 1156-1162.

[11] Milin-Sipus, Z., Ruled Weingarten surfaces in Galilean Space, Period. Math. Hung., 56(2008), 213-225. 
[12] Milin-Sipus, Z., Divjak, B., Some special surface in the pseudo-Galilean space, Acta Math. Hungar., 118(2008), 209-226.

[13] Ogrenmiş, A.O., Bektaş, M., Ergüt, M., On helices in the Galilean space $G_{3}$, Iranian Journal of Science \& Technology, Transaction A, 31(A02)(2007), 177-181.

[14] Ogrenmiş, A.O., Ŏztekin, H., Ergüt, M., Bertrand curves in Galilean space and their characterizations, Kragujevac J. Math., 32(2009), 139-147.

[15] Pavkovic, B.J., The general solution of the Frenet system of differential equations for curves in the Galilean space $G_{3}$, Rad Hazu Math., 450(1990), 123-128.

[16] Pavkovic, B.J., Kamenarovic, I., The equiform differential geometry of curves in the Galilean space, Glasnik Matematicki, 22(1987), 449-457.

[17] Roschel, O., Die Geometrie des Galileischen Raumes, Habilitationsschrift, Leoben, 1984.

[18] Sahin, T., Yilmaz, M., the rectifying developable and the tangent indicatrix of a curve in Galilean 3-space, Acta Math. Hungar., 132(2011), no. 1-2, 154-167.

[19] Sorour, A.H., Weingarten Tube-like Surfaces in Euclidean 3-Space, Stud. Univ. BabeşBolyai Math., 61(2016), no. 2, 239-250.

[20] Suk Ro, J., Yoon, D.W., Tubes of Weingarten types in a Euclidean 3-space, J. Chungcheong Math. Soc., 22(2009), 359-366.

[21] Xu, Z., Feng, R., Sun, J., Analytic and algebraic properties of canal surfaces, J. Comput. Appl. Math., 195(2006), 220-228.

Adel H. Sorour

Department of Mathematics, Faculty of Science

Sohag University, 82524, Sohag, Egypt

e-mail: adel7374@yahoo.com 